\title{
THE USE OF ARTIFICIAL INTELLIGENCE IN RADIOLOGICAL DIAGNOSIS AND DETECTION OF DENTAL CARIES: A SYSTEMATIC REVIEW
}

\author{
Karolina Futyma-Gąbka (D), Ingrid Różyło-Kalinowska (D) \\ Department of Dental and Maxillofacial Radiodiagnostics, Medical University of Lublin, Poland
}

\begin{abstract}
Dental caries is a very common condition, which can lead to serious complications, including tooth loss and infection of the whole human body. Dentists in their daily practice, apart from visual-tactile examination, use radiological methods, such as periapical radiographs and bitewings. Artificial intelligence (AI) is a tool that can be used in diagnosing and detecting cavities. It can help to avoid more invasive treatment and further consequences. The goal of this systematic review was to present the use of artificial intelligence in radiological dental caries diagnostics. In total, twelve studies meeting inclusion criteria were analyzed, and image databases varied from 93 to 3,868 radiographs, with average value of 1,091.17 radiographs. Most of the included studies employed bitewings and periapical images, and authors used different methods and AI algorithms. Accuracy was performed in nine researches. The highest accuracy was $99 \%$, the lowest $73.3 \%$. Also, nine researches provided information on number of observers, which varied from 1 to 25 . Comparing all the studies, it was difficult to draw out a conclusion. Artificial intelligence in radiological images may assist dentists and radiologist to perform better and faster examination, and it may be a used in routine dental care. However, more researches are needed in the field of dentistry and radiology.
\end{abstract}

KEY WORDS: artificial intelligence, dental caries, radiology, detecting caries.

J Stoma 2021; 74, 4: 262-266

DOI: https://doi.org/10.5114/jos.2021.111664

\section{INTRODUCTION}

Artificial intelligence (AI) is a technology that is capable of learning and using knowledge to perform specific tasks [1]. In medicine, AI tools are being used for self-detection of pathologies, prediction of possibility of disease occurrence, and evaluation of prognosis. Many studies have reported promising conclusions about $\mathrm{AI}$ in automatic detection of coronary artery calcification [2], cerebral micro-bleeds [3], diabetic retinopathy [4], and breast or skin cancer [5-7]. This tool may have a potential to identify image changes, which are not easily perceived by humans. For example, machine analysis of brain magnetic resonance imaging (MRI) can detect tissue changes in early ischemic stroke with higher sensitivity than a human expert [8]. In dentistry, machine learning models being part of artificial intelligence, can be useful to diagnose periapical cysts, granuloma, bone cancer, stages of osteoporosis, periodontal inflammation, or dental caries $[9,10]$. Machine learning seems to be a very powerful component of AI. Recently, convolutional neural networks (CNNs) have shown very promising results in many fields of medicine and dentistry [10]. Their algorithms provide structures, shapes,

\section{JOURNAL OF} STOMATOLOGY CZASOPISMO STOMATOLOGICZNE

AdDRESS FOR CORRESPONDENCE: Karolina Futyma-Gąbka,

Department of Dental and Maxillofacial Radiodiagnostics, Medical University of Lublin, Poland, e-mail: lek.dent.karolina.futyma@gmail.com

ReCEIVED: 20.09.2021 • ACCEPTED: 15.11.2021 • Published: 30.11.2021 
and patterns in a process of 'training' without human involvement. This technique, besides detection of lesions, can be helpful in evaluation of patient survival, for example with glioblastomas $[11,12]$. CNN consists of many layers. When the input image is analyzed, hidden layers usually bring out and combine features into feature maps, and fully connected layers provide advanced reasoning. Finally, an output layer produces predictions [13]. Depending on type of task, the application delivers outcome presented with a unique label (caries or without caries) or a list of different labels (teeth names/numbers) [8]. Various studies have reported that AI systems can enhance accuracy of diagnosis, assist in treatment planning, and reduce time needed for evaluation [13].

Dental caries is one of the most frequent infectious diseases all over the world [14-16]. Fast and correct diagnosis is essential for effective treatment. There are many different methods for detecting tooth decay, including visual-tactile inspection, fiber optic transillumination (FOTI), and devices based on fluorescence and radiography [16]. However, none of those provide $100 \%$ accuracy.

Most of dentists use at least two complementing methods, especially visual-tactile examination with periapical X-rays or bitewings [17]. However, even experienced dentist can miss cavities, mostly in the interproximal area, with $20-40 \%$ probability [18]. One of the most common tasks in dentists practice is to detect early caries lesions to provide treatment preventing more serious complications [17]. In order to improve accuracy in diagnostics, recent computer-aided systems have been studied. This review aims to evaluate studies investigating radiological caries diagnosis and its' detection with artificial intelligence technology.

\section{MATERIAL AND METHODS}

The main research question was elaborated considering PICO's elements (Table 1). An electronic search was conducted using the following databases: PubMed, ScienceDirect, and Institute of Electrical and Electronics Engineers (IEEE) Xplore. Key words, included 'artificial intelligence', 'radiology', and 'dental caries' in different combinations. Records were collated and titles were screened for duplicates. Bibliographic search was performed and articles, which met inclusion criteria

TABLE 1. Description of PICO's elements used during the search

\begin{tabular}{|l|l|}
\hline Criteria & Specification \\
\hline P (population) & Radiological images obtained from human dental region \\
\hline I (intervention) & Dental caries diagnostic model based on Al algorithms \\
\hline C (comparator) & Reference standard (expert's judgement, etc.) \\
\hline O (outcome) & $\begin{array}{l}\text { Outcome metrics and values (accuracy, sensitivity, } \\
\text { specificity, etc.) }\end{array}$ \\
\hline
\end{tabular}

were selected. Inclusion criteria for the studies were: full manuscripts published in English, including conference proceedings and radiology-based studies with AI models for diagnosis of dental caries. Exclusion criteria were as follows: abstracts, review articles, book chapters, and other studies using AI models to detect caries lesions without radiological component. Also, not available or accessible full-text papers were excluded. There was no limit on publication period.

Each study was analyzed by publication year, type of image, total image database, number of examiners, type of AI tool employed to detect caries, and whether given database had been modified and improved before training process. Subsequently, type of teeth, examined surfaces, and outcome metrics values were analyzed.

\section{RESULTS}

Initially, a total of 238 titles were identified. After excluding duplicates, reviews, abstracts, book chapters, and title screening, 21 articles were approved for full-text reading. Then, inclusion criteria evaluation was performed, and 12 articles were included into analysis (Figure 1).

Included studies were conducted and published between 2008 and 2021. The most used type of radiographic image were bitewings $(n=5)$ and periapical $\mathrm{X}$-rays $(n=5)$. Other methods, such as panoramic X-rays $(n=1)$ and cone-beam computed tomography (CBCT) $(n=1)$ were not common. The total image databases varied from 93 to 3,868 images, with a mean of 1,091.17 images. Nine articles consisted of information about number of observers (dentists/radiologists).

The highest number of observers was 25 , whereas in two papers, there was only 1 examiner and in three articles, no information was provided about number of people involved in evaluation. Of those 9 studies, only 4 included level of experience of observers that varied

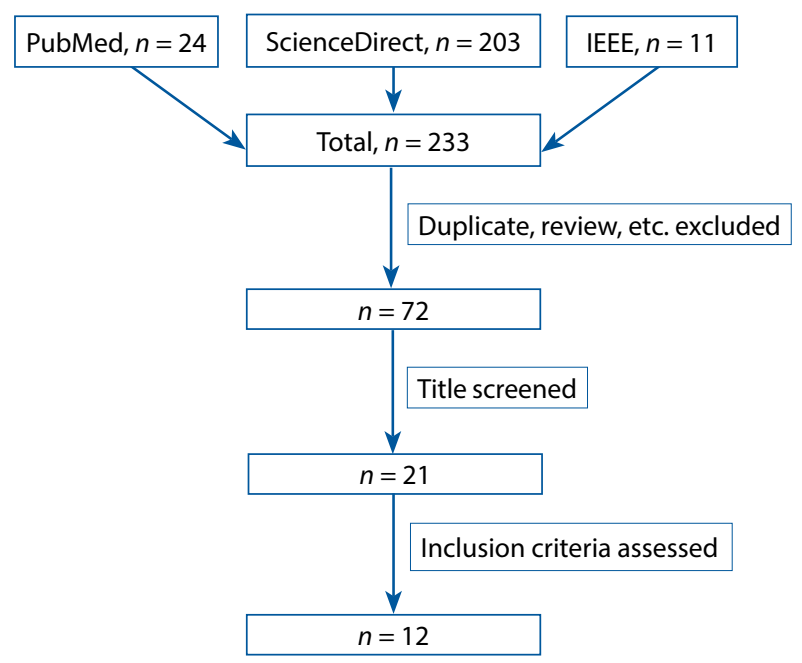

FIGURE 1. Flowchart illustrating the study selection process 
from 3 to more than 20 years. All of the studies explained what AI model was used, but in four papers, no information was given on pre-processing of images from database. Four studies described in detail teeth taken into account, and also four studies distinguished surface of the considered tooth. In all the investigated papers, it was the approximal surface. The accuracy was provided in nine articles. The highest accuracy was $99 \%$, and the lowest $73.3 \%$. Other metrics values were specificity, sensitivity, positive prediction value (PPV), negative prediction value (NPV), receiver operating characteristic
(ROC), Matthews correlation coefficient (MCC), intraclass correlation coefficient (ICC), area under curve (AUC), precision recall curve (PRC area), F1 score, etc. Some of the researchers reported on information about numbers of layers in CNN algorithms (for example, in Lee et al., 22 layers in Inception-v3 architecture) [19]. In one of the studies, apart from evaluation of accuracy of CNN, inter-observer consistency (ICC) was evaluated, and required diagnostic time for two groups of examiners, unaided and AI-aided, was compared. Details of all included studies are shown in Tables 2 and 3.

TABLE 2. Details of all included studies

\begin{tabular}{|l|c|c|c|c|c|}
\hline \multicolumn{1}{|l|}{ Study [Ref.] } & \multicolumn{1}{c}{ Year of publication } & \multicolumn{1}{c|}{ Type of image } & \multicolumn{1}{c|}{ Total image database } & Number of examiners & Examiners clinical experience \\
\hline Bayraktar [21] & 2021 & Bitewings & 1,000 & 2 & At least 10 years \\
\hline Cantu [24] & 2020 & Bitewings & 3,686 & 7 & $3-14$ years \\
\hline Lee [19] & 2018 & Periapical & 3,000 & 4 & - \\
\hline Srivastava [25] & 2017 & Bitewings & 3,000 & 3 & - \\
\hline Ezhov [13] & 2021 & CBCT & 1,346 & 24 & At least 5 years \\
\hline Geetha [26] & 2020 & Periapical & 105 & 1 & - \\
\hline Devito [20] & 2008 & Bitewings & 160 & 25 & More than 20 years \\
\hline Valizadeh [18] & 2015 & Periapical & 221 & 1 & - \\
\hline Singh [29] & 2017 & Panoramic radiographs & 93 & - & - \\
\hline Moran [28] & 2021 & Bitewings & 112 & 3 & - \\
\hline Sornam [30] & 2017 & Periapical & 120 & - & - \\
\hline Prajapati [27] & 2017 & Periapical & 251 & - & - \\
\hline
\end{tabular}

TABLE 3. Type of teeth, Al model, and outcome metrics in examined studies

\begin{tabular}{|c|c|c|c|}
\hline Study [Ref.]. & Type of teeth & Al model & Outcome metrics \\
\hline Bayraktar [21] & Premolars, molars, and both & YOLO-based CNN with holdout validation & $\begin{array}{c}\text { Accuracy, sensitivity, specificity, PPV, } \\
\text { NPV, and AUC }\end{array}$ \\
\hline Cantu [24] & - & CNN, U-Net-architecture & $\begin{array}{c}\text { Accuracy, sensitivity, specificity, PPV, } \\
\text { NPV, F1, and MCC }\end{array}$ \\
\hline Lee [19] & Premolars, molars, and both & CNN, Inception-v3 architecture & $\begin{array}{l}\text { Accuracy, sensitivity, specificity, PPV, } \\
\text { NPV, ROC curve, and AUC }\end{array}$ \\
\hline Srivastava [25] & - & FCNN (deep fully convolutional neural network) & Recall, precision, and F1 score \\
\hline Ezhov [13] & - & CNN, U-Net-architecture (Diagnocat) & Sensitivity, specificity, and ICC \\
\hline Geetha [26] & - & ANN with 10 -fold cross validation & $\begin{array}{l}\text { Accuracy, false positive, ROC area, } \\
\text { and precision recall curve (PRC area) }\end{array}$ \\
\hline Devito [20] & Extracted premolars and molars & Multilayer perceptron neural network & ROC \\
\hline Valizadeh [18] & Extracted premolars and molars & Fuzzy c-means algorithm (FCM) & ICC (intraclass correlation coefficient) \\
\hline Singh [29] & - & $\begin{array}{l}\text { Radon transformation (RT) and discrete cosine } \\
\text { transformation (DCT) }\end{array}$ & Accuracy \\
\hline Moran [28] & - & CNN, inception, and ResNet architectures & $\begin{array}{c}\text { Accuracy, sensitivity, specificity, PPV, } \\
\text { NPV, AUC, and ROC }\end{array}$ \\
\hline Sornam [30] & - & Feedforward neural network & Accuracy \\
\hline Prajapati [27] & - & CNN & Accuracy \\
\hline
\end{tabular}




\section{DISCUSSION}

There are various indications to employ AI models in medicine and dentistry. The main goal of the present review was to analyze the use and performance of artificial intelligence applications in dental caries detection. However, there are few studies on this issue. Some of them have attempted to develop detection models by using images of extracted teeth $[18,20]$. These two articles used as a gold standard histological examination (microscope), while in other studies, human experts performed evaluation before dataset training. The remaining studies present some limitations, because when the neural network is trained with dentists or radiologists, the system relies on their experience and the output depends on the quality of the input. Most of the studies analyzed bitewings or periapical radiographs, which are common tools in everyday dentistry. Bayraktar et al. [21] used database consisting of 1,000 bitewings to evaluate accuracy of diagnosis of inter-proximal caries lesions. A system, YOLO-based CNN yielded $94.59 \%$ accuracy. Promising results show that studies in this area can allow for more accurate and potentially faster caries diagnosis. It could also assist dentists with less clinical experience or improve clinical education of dental students.

Ezhov et al. [13] used a novel system called Diagnocat based on CBCT images, consisting of 5 modules: ROI localization, tooth localization and numeration, caries localization, periodontitis, and periapical lesion localization module. Authors, apart from many other measures, also examined inter-observer consistency (ICC), and for caries detection, it was not statistically significant ( $p$-value less than 0.05). In the second part of their research, they evaluated 30 CBCTs divided into two groups: unaided and AI-aided. Statistical tests revealed that using Diagnocat application, superior influence on improving sensitivity in dental diagnosis was observed. Additionally, the average time needed to evaluate an image in real-time clinical environment was measured, and for aided group, the time was 17.55 minutes, while it took 18.74 minutes for the unaided one. Statistical tests revealed that the AI-aided group had a shorter assessment period in comparison with unaided group $(p=0.032)$. However, caries detection was not a basic indication for a CBCT examination. Nevertheless, when performing scans due to other clinical purposes, experts should also evaluate for the presence/absence of cavities.

Deep learning, especially with a $\mathrm{CNN}$, is gaining attention because of its' high accuracy in image evaluation. $\mathrm{CNN}$ algorithm is based on physiological mechanism of recognition at primary visual cortex of the vertebrate (neocognitron) [22]. With deep learning, pattern recognition of images can be provided without human experts. This technique has a potential to perform correlation between radiological data and clinical information [12].

However, overfitting is the major problem that deep learning has to overcome. It means that an artificial in- telligence model has learned a way to evaluate training sample, and it may be no longer applicable to general population. The most effective way to face the problem of overfitting is to collect more training data [23].

As mentioned above, databases used for AI caries studies varied from 93 to 3,868 cases. Three publications with the highest number of radiographic images seem to be the most dependable one. Cantu et al. [24] accessed 3,686 bitewings dataset, and 3,293 of them were used for training. AI model was $\mathrm{CNN}, \mathrm{U}-\mathrm{Net}$-architecture, with Intersection-over-Union as validation metric. The accuracy of this application was 0.80 , while in human observers was 0.71 with low sensitivity, especially for initial lesions. Lee et al. conducted a research with a large image base [19], with 3,000 periapical radiographs, including 2,400 used for training and validation and 600 for evaluation (test). The authors used CNN, GoogleNet, and Inception-v3 architecture consisting of 22 layers that showed 82.0 accuracy in detection and diagnosis of dental caries. Srivastava et al. [25] used individually created CNN model, FCNN (deep fully convolutional neural network), with more than 100 layers on 3,000 bitewings, and 2,500 samples for training and 500 for testing. The outcome metric values showed recall (sensitivity) of 80.5 , precision (positive predictive value) of 61.5 , and F1-score (agreement with truth) of 70. However, owing to application of different $\mathrm{CNN}$ architectures, it was not possible to compare these three studies.

Studies [26-30] with the smallest database, excluding those on extracted teeth (with microscope assessment), seems to have lower reliability. The accuracy values varied from $73.3 \%$ to $99 \%$, and one with the highest accuracy used FeedForward Neural Network, and measured only this outcome metric.

Recently, promising results were published by Schwenficke et al. [31], who assessed cost-effectiveness of AI for caries detection in bitewings. Effectiveness was measured as the average time of keeping a tooth in the oral cavity. One of the conclusions was that this cost-effectiveness can be modified by a risk profile of the population. In highrisk populations, AI model was more sensitive than in low-risk populations.

The main limitation of this systematic review was the small number of available publications. Unfortunately, due to large discrepancies between AI methods, it was difficult to accurately compare all the studies. It is necessary to standardize criteria, such a golden standard, observers experience, type of image, and outcome metrics to precisely confront the accuracy of different AI methods in detection and diagnosis caries lesions. It is certain that artificial intelligence and its' variety of algorithms has an influence on modern medicine and dentistry. It can enhance accuracy of diagnosis, reduce time required for examination, facilitate treatment planning, and avoid invasive treatment methods. Development of these techniques requires human experts to become familiar with using this powerful tool. 


\section{CONCLUSION}

The use of artificial intelligence in diagnosis of dental caries seems promising, but more research is required to provide solid evidence.

\section{CONFLICT OF INTEREST}

The authors declare no potential conflicts of interest with respect to the research, authorship, and/or publication of this article.

\section{References}

1. Stone P, Brooks JR, Brynjolfsson E, et al. Artificial intelligence and life in 2030. One hundred year study on artficial intelligence: report of the 2015-2016 study panel, Standford University, Standford, CA. DOI: https://ai100.standford.edu/2016-report.

2. van Velzen SGM, Lessmann N, Velthuis BK, et al. Deep learning for automatic calcium scoring in CT: validation using multiple cardiac CT and chest CT protocols. Radiology 2020; 295: 66-79.

3. van den Heuvel TL, van der Eerden AW, Manniesing R, et al. Automated detection of cerebral microbleeds in patients with traumatic brain injury. Neuroimage Clin 2016; 12: 241-251.

4. Gulshan V, Peng L, Coram M, et al. Development and validation of a deep learning algorithm for detection of diabetic retinopathy in retinal fundus photographs. JAMA 2016; 13: 2402-2410.

5. Hosny A, Parmar C, Quackenbush J, Schwartz LH, Aerts HJWL Artificial intelligence in radiology. Nat Rev Cancer 2018; 18: 500-510.

6. Esteva A, Kuprel B, Novoa R, et al. Dermatologist-level classification of skin cancer with deep neural networks. Nature 2017; 2: 115-118.

7. Hung K, Yeung AWK, Tanaka R, Bornstein MM. Current applications, opportunities, and limitations of AI for 3D imaging in dental research and practice. Int J Environ Res Public Health 2020; 17: 4424 .

8. Oren O, Gersh BJ, Bhatt DL. Artificial intelligence in medical imaging: switching from radiographic pathological data to clinically meaningful endpoints. Lancet Digital Health 2020; 2: e486-488.

9. Hung K, Montalvao C, Tanaka R, Kawai T, Bornstein MM. The use and performance of artificial intelligence applications in dental and maxillofacial radiology: a systematic review. Dentomaxillofac Radiol 2020; 49: 20190107. DOI: 10.1259/dmfr.20190107.

10. Schwendicke F, Golla T, Dreher M, Krois J. Convolutional neural networks for dental image diagnostics: a scoping review. J Dent 2019; 91: 103226. DOI: 10.1016/j.jdent.2019.103226.

11. Lao J, Chen Y, Li ZC, et al. A deep learning-based radiomics model for prediction of survival in glioblastoma multiforme. Sci Rep 2017; 7: 10353. DOI: 10.1038/s41598-017-10649-8

12. Yasaka K, Akai H, Kunimatsu A, Kiryu S, Abe O. Deep learning with convolutional neural network in radiology. Jpn J Radiol 2018 36: 257-272.

13. Ezhov M, Gusarev M, Golitsyna M. Clinically applicable artificial intelligence system for dental diagnosis with CBCT. Sci Rep 2021; 11: 15006. DOI: 10.1038/s41598-021-94093-9.

14. Featherstone JD. The science and practice of caries prevention. J Am Dent Assoc 2000; 131: 887-899.

15. Islam B, Khan SN, Khan AU. Dental caries: from infection to prevention. Med Sci Monit 2007; 13: 196-203.

16. Gomes J. Detection and diagnosis of the early caries lesion. BMC Oral Health 2015; 15 (Suppl 1): S3. DOI: 10.1186/1472-6831-15-S1-S3.

17. Schwendicke F, Tzschoppe M, Paris S. Radiographic caries detection: a systematic review and meta-analysis. J Dent 2015; 43 924-933.

18. Valizadeh S, Goodini M, Ehsani S, Mohseni H, Azimi F, Bakhshandeh H. Designing of a computer software for detection of approxi- mal caries in posterior teeth. Iran J Radiol 2015; 12: e16242. DOI: 10.5812/iranjradiol.12(2)2015.16242.

19. Lee JH, Kim DH, Jeong SN, Choi SH. Detection and diagnosis of dental caries using a deep learning-based convolutional neural network algorithm. J Dent 2018; 77: 106-111.

20. Lopes Devito K, de Souza Barbosa F, Filho WNF. An artificial multilayer perceptron neural network for diagnosis of proximal dental caries. Oral Surg Oral Med Oral Pathol Oral Radiol Endod 2008; 106: 879-884.

21. Bayraktar Y, Ayan E. Diagnosis of interproximal caries lesions with deep convolutional neural network in digital bitewing radiographs. Clin Oral Investig 2021. DOI: 10.1007/s00784-021-04040-1 [Online ahead of print].

22. Fukushima K, Miyake S. Neocognitron: a new algorithm for patern recognition tolerant of deformations and shift in position. Pattern Recognition 1982; 15: 455-469.

23. Mutasa S, Sun S, Ha R. Understanding artificial intelligence based radiology studies: what is overfitting? Clin Imaging 2020; 65: 96-99.

24. Cantu AG, Gehrung S, Krois J, et al. Detecting caries lesions of different radiographic extension on bitewings using deep learning. J Dent 2020; 100: 103425. DOI: 10.1016/j.jdent.2020.103425.

25. Srivastava MM, Kumar P, Pradhan L, Varadarajan S. Detection of tooth caries of bitewing radiographs using deep learning. arXiv: 1711.07312 [cs.CV]; 2017.

26. Geetha V, Aprameya KS, Hinduja DM. Dental caries diagnosis in digital radiographs using back-propagation neural network. Health Inf Sci Syst 2020; 8: 8-14.

27. Prajapati SA, Nagaraj R, Mitra S. Classification of dental diseases using $\mathrm{CNN}$ and transfer learning. In Proceedings of the $5^{\text {th }}$ International Symposium on Computational and Business Intelligence (ISCBI), Dubai, UAE, 2017; 70-74

28. Moran M, Faria M, Giraldi G, Bastos L, Oliveira L, Conci A. Classification of approximal caries in bitewing using convolutional neural network. Sensors (Basel) 2021; 21: 5192. doi: 10.3390/ s21155192.

29. Singh P, Sehgal P. Automated caries detection based on Radon transformation and DCT. $20178^{\text {th }}$ International Conference on Computing, Communication and Networking Technologies (ICCCNT). Dehli, 2017; 1-6.

30. Sornam M, Prabhakaran M. A new linear adaptive swarm intelligence approach using back propagation neural network for dental caries classification. 2017 IEEE International Conference on Power, Control, Signals and Instrumentation Engineering (ICPCSI) DOI: 10.1109/ICPCSI.2017.8392208.

31. Schwenficke F, Rossi JG, Göstemeyer G, et al. Cost-effectiveness of artificial intelligence for proximal caries detection. J Dent Res 2021; 100: 369-376 